\title{
Gastroprotective Mechanism and Ulcer Resolution Effect of Cyrtocarpa procera Methanolic Extract on Ethanol-Induced Gastric Injury
}

\author{
Wendy Itzel Escobedo-Hinojosa, ${ }^{1}$ Erika Gomez-Chang $\mathbb{D}^{1},{ }^{1}$ Karina García-Martínez, \\ Raquel Guerrero Alquicira, ${ }^{2}$ Alexandre Cardoso-Taketa, ${ }^{3}$ and Irma Romero ${ }^{1}{ }^{1}$ \\ ${ }^{1}$ Departamento de Bioquímica, Facultad de Medicina, Universidad Nacional Autónoma de México, Ciudad Universitaria, \\ 04510 Ciudad de México, Mexico \\ ${ }^{2}$ Departamento de Biología Celular y Tisular, Facultad de Medicina, Universidad Nacional Autónoma de México, \\ Ciudad Universitaria, 04510 Ciudad de México, Mexico \\ ${ }^{3}$ Centro de Investigación en Biotecnología, Universidad Autónoma del Estado de Morelos, Av. Universidad 1001, \\ Col. Chamilpa, 62209 Cuernavaca, MOR, Mexico
}

Correspondence should be addressed to Irma Romero; irma@bq.unam.mx

Received 29 August 2017; Accepted 29 November 2017; Published 9 January 2018

Academic Editor: Vincenzo De Feo

Copyright (C) 2018 Wendy Itzel Escobedo-Hinojosa et al. This is an open access article distributed under the Creative Commons Attribution License, which permits unrestricted use, distribution, and reproduction in any medium, provided the original work is properly cited.

\begin{abstract}
Gastric ulcers are a worldwide health problem and their poor healing is one of the most important causes for their recurrence. We have previously reported the remarkable gastroprotective and anti-Helicobacter pylori activities of the methanolic extract (CpMet) of Cyrtocarpa procera bark. This work investigates, in a murine model, the CpMet gastroprotective mechanism and establishes its preclinical efficacy in the resolution of ethanol-induced gastric ulcers. The results showed that the gastroprotective activity of CpMet is mainly associated with endogenous $\mathrm{NO}$ and prostaglandins, followed by sulfhydryl groups and $\mathrm{K}_{\text {ATP }}$ channels. Furthermore, CpMet $(300 \mathrm{mg} / \mathrm{kg}$, twice a day) orally administered during 20 consecutive days promoted an ulcer area reduction of $62.65 \%$ at the 20th day of the treatment. The effect was confirmed macroscopically by the alleviation of gastric mucosal erosions and microscopically by an increase in mucin content and a reduction in the inflammatory infiltration at the site of the ulcer. No clinical symptoms or signs of toxicity were observed in the treated animals. The results indicate the safety and efficacy of CpMet in promoting high quality of ulcer healing by different mechanisms, but mostly through cytoprotective and anti-inflammatory effects, making it a promising phytodrug for ulcer treatment.
\end{abstract}

\section{Introduction}

Gastric ulcers (GU) are open sores in the lining of the stomach that extend to or beyond the muscularis mucosa. The incidence of GU varies widely around the world depending on the age, gender, and geographical location but they remain a very common condition worldwide and a major public health problem due to high healthcare costs and mostly to life-threatening complications such as bleeding, perforation, and obstruction, which explains the high morbidity and mortality associated with this disease [1-4].

The pathophysiology of gastric ulceration is multifactorial but is generally considered as a result of an imbalance in the equilibrium between protective and aggressive factors of the gastric mucosa [5]. The gastrointestinal defense mechanisms include gastric mucosal integrity, mucus secretion, bicarbonate production, nitric oxide (NO), gastroprotective prostaglandin synthesis, normal gastric motility, and adequate tissue microcirculation, while the noxious factors comprise, among others, gastric acid and pepsin secretion, bile salts, reactive oxygen species (ROS), Helicobacter pylori infection, alcohol consumption, and prolonged ingestion of nonsteroidal anti-inflammatory (NSAIDs) drugs [6-8].

As expected, the current treatments of GU are targeted for either enhancing gastric mucosal defenses or counteracting 
injurious factors. The hallmark drugs have been the ones that reduce gastric acid secretion such as $\mathrm{H}_{2}$-receptor antagonists (e.g., ranitidine) and proton pump inhibitors (e.g., omeprazole) as well as antibiotic therapy for $H$. pylori eradication [3]. Despite the fact that acid antisecretory drugs have been a cornerstone in the treatment of this pathology, the high costs and side effects of long-term regimens combined with ulcer recurrence and some cases of refractoriness to conventional acid suppression therapies urge to search for new antiulcer agents addressed to enhance the healing of GU with fewer disadvantages than current treatments $[9,10]$. The quality of ulcer healing $(\mathrm{QOUH})$ is a key point in the pathogenesis of gastric ulcers since it has been reported that abnormalities in mucosal regeneration within the scars of healed ulcers, as well as the persistence of chronic inflammation demonstrated by the presence of increased infiltration of neutrophils and macrophages, are the basis for ulcer recurrence [11]. Therefore, the research of new therapeutic agents should also focus on improving the QOUH. In this sense, herbal drugs have become excellent sources for the development of new treatments to heal GU since they are effective, reduce the offensive factors, appear to be safer, have better tolerance in patients, and are less expensive for the populations $[12,13]$.

Cyrtocarpa procera Kunth (Anacardiaceae) commonly known as "chupandilla," "copalcojote," or "coco de cerro" is endemic to Mexico. It is a tree mainly distributed in the deciduous and subdeciduous dry forests of the states of Jalisco, Michoacán, Nayarit, Guerrero, Oaxaca, México, Morelos, and Puebla. The aqueous decoction and infusion of $C$. procera bark have been extensively used in Mexican folk medicine for digestive disorders such as dysentery and diarrhea, for kidney ailments, and for toothaches, among other uses [14-16]. Due to its similar appearance, $C$. procera bark is used to adulterate the Amphipterygium adstringens bark, one of the most important and commercialized Mexican medicinal plants used to treat gastritis, gastric ulcer, and stomach cancer [14, 17].

Only few studies have addressed the phytochemical and pharmacological properties of $C$. procera bark. Phytochemical studies have reported the isolation and identification of some sterols such as $\beta$-amyrin and $\beta$ sitosterol, and the fatty acids 1,3-propyl-dipentadecanoate, 3-hydroxypropyl-9-octadecenoate, pentadecylbenzene, eicosylbenzene, docosane, heptacosane, dotriacontane, and 2,6,10-trimethyltetradecane [18, 19]. Concerning the pharmacological information, it has been explored the antiulcerogenic activity of $C$. procera bark extracts in ethanol-induced gastric ulcers in a rat model [19] and the antibiotic activity of a methanolic extract against a range of Gram-positive and Gram-negative bacteria [15]. In the latter case, our group has reported the anti-Helicobacter pylori activity of aqueous and methanolic extracts of $C$. procera [20].

In our previous work with this plant [21] we made a systematic evaluation of the anti- $H$. pylori, anti-inflammatory and gastroprotective properties of different polarity C. procera bark extracts in order to identify if any of them had polypharmalcological effects. The results demonstrated a remarkable gastroprotective activity of the methanolic extract $\left(\mathrm{ED}_{50}=0.53 \mathrm{mg} / \mathrm{kg}\right)$ in an acute ethanol ulcer model and a good in vitro anti-H. pylori activity $(\mathrm{MIC}=62.5 \mu \mathrm{g} / \mathrm{ml})$ being more effective than the reference antibiotic metronidazole. Also, the methanolic extract was not toxic under acute administration and had high yield, making it a good candidate for further clinical studies.

Thus, in order to further clarify the efficacy of the methanolic extract of C. procera (CpMet) as an antiulcerative agent, the present work was undertaken to investigate the gastroprotective mechanism and to establish its preclinical efficacy in the resolution of gastric ulcers induced by ethanol as well as its toxicological safety during a 20-day repeateddose oral administration.

\section{Materials and Methods}

2.1. Plant Material. The collection of C. procera stem bark was made in Jojutla, Morelos, in the Higueron Locality $\left(18^{\circ} 37^{\prime} 12.5^{\prime \prime} \mathrm{N}, 99^{\circ} 10^{\prime} 33.7^{\prime \prime} \mathrm{W}\right)$, on March 2010 , and it was identified by Mtra. Rosa María Fonseca from the Faculty of Sciences, Universidad Nacional Autónoma de México (UNAM). The species vouchers specimens 125630 and 125636 were deposited in the Faculty of Sciences Herbarium, UNAM. The name of the plant has been checked as an accepted name on http://www.theplantlist.org (accessing date 26/08/17).

2.2. Preparation of Methanolic Extract. The methanolic extract (CpMet) was prepared by exhaustive maceration of $1.5 \mathrm{~kg}$ of dried milled vegetable material with methanol $(1: 10 \mathrm{w} / \mathrm{v})$. The solvent was separated from the residue by filtration and then evaporated to dryness under reduced pressure. $431 \mathrm{~g}$ of CpMet, equivalent to $28.7 \%$ of dry weight yield, was obtained. The extract was kept at room temperature in the dark until it was used.

2.3. Animals. Male CD-1 mice from UNAM's Faculty of Medicine vivarium weighting 20-25g were used for the toxicity evaluation and 35-40 $\mathrm{g}$ mice for the rest of the experiments. They were housed in standard laboratory conditions ( $22 \pm 2^{\circ} \mathrm{C}, 12$-h light dark cycle), with free access to standard pellet diet (Rodent Lab Chow Purina ${ }^{\circledR}$ ) and water ad libitum. 12 hours prior to each experiment, the animals were deprived of food with free access to water and placed in individual wire-net raised floors to prevent coprophagy. The experimental protocols were approved by the Ethical Committee of the UNAM's Faculty of Medicine (approval number 087/2013) and conducted in conformity with the Mexican Official Norm for animal care and handling (NOM-062-ZOO-1999) and in accordance with the ethical guidelines of the National Institutes of Health. The assays recorded in this work required different number of animals as indicated below.

2.4. Chemicals and Drugs. The methanolic extract of $C$. procera was dissolved in $0.9 \% \mathrm{NaCl}$ (isotonic saline solution). Absolute ethanol (ETOH), $\mathrm{N}^{\omega}$-nitro-L-arginine methyl ester (L-NAME), N-ethylmaleimide (NEM), glibenclamide (GLIB), indomethacin (INDO), and carbenoxolone (CAR) were purchased from Sigma-Aldrich Co. Omeprazole (OME) was obtained from Liomont ${ }^{\circledR}$. Ketamine and Xylazine were acquired from Pisa ${ }^{\circledR}$. 
All the samples administered by intragastric route, independently of the final concentration used, were always suspended in a volume of $10 \mathrm{ml} / \mathrm{kg}$.

\subsection{Ethanol-Induced Gastric Mucosal Lesions in $N^{\omega}$-Nitro-} L-arginine Methyl Ester, Indomethacin, N-Ethylmaleimide, and Glibenclamide-Pretreated Mice. In order to investigate the CpMet gastroprotective mechanism of action, separate experiments were conducted using the following drugs to pretreat the animals: INDO, a prostaglandin synthesis inhibitor $(10 \mathrm{mg} / \mathrm{kg}$ dissolved in $5 \mathrm{mM} \mathrm{NaHCO}$, s.c.); $\mathrm{NEM}$, a sulfhydryl compound blocker $(1 \mathrm{mg} / \mathrm{kg}$ dissolved in isotonic saline solution, s.c.); L-NAME, a nitric oxide synthase inhibitor $(10 \mathrm{mg} / \mathrm{kg}$ dissolved in isotonic saline solution, i.p.); and GLIB, an ATP-sensitive potassium channel blocker ( $5 \mathrm{mg} / \mathrm{kg}$ dissolved in isotonic saline solution, i.g.). The corresponding vehicle, either $5 \mathrm{mM} \mathrm{NaHCO}_{3}$ or isotonic saline solution, was administered in control groups. The animal groups $(n=6)$ received the pretreatments $30 \mathrm{~min}$ before the intragastric administration of the respective treatment, either isotonic saline solution $(10 \mathrm{ml} / \mathrm{kg})$ or CpMet $(100 \mathrm{mg} / \mathrm{kg})$. An hour later, the ulceration was induced according to the method described by Bucciarelli and Skliar [22] by intragastric instillation of ETOH at a dose of $7 \mathrm{ml} / \mathrm{kg}$ of body weight. An hour and a half after ETOH administration, animals were sacrificed by carbon dioxide inhalation in an appropriate chamber. Each stomach was dissected out, insufflated with $2 \mathrm{ml}$ of $10 \%$ formalin, and fixed in the same solution for $15 \mathrm{~min}$. The stomachs were opened along the greater curvature, pressed between two glass plates, and scanned. The lesion area was determined with the aid of a public domain Java image processing program (Image J) developed at the US National Institutes of Health (freely available at https://rsb.info.nih.gov/nih-image/). The sum of the areas of all ulcers in the corpus of each stomach was considered as the lesion area $\left(\mathrm{mm}^{2}\right)$. At least three independent experiments were performed.

2.6. pH Measurement of Gastric Contents. In order to determine whether the oral administration of CpMet modifies the $\mathrm{pH}$ value of gastric contents, the following assay was performed according to the method described by Shay et al. [23] with modifications. Briefly, after $12 \mathrm{~h}$ of fasting, the animals were separated into different groups $(n=6)$ and the treatments (CpMet $100 \mathrm{mg} / \mathrm{kg}$; omeprazole $20 \mathrm{mg} / \mathrm{kg}$ ) or the vehicle $(10 \mathrm{ml} / \mathrm{kg})$ was administrated by oral gavage. One hour later, mice were anesthetized with ketamine/xylazine $(150 / 10 \mathrm{mg} / \mathrm{kg}$, i.p.). The abdomen was opened through a midline epigastric incision, and the pyloric portion was occluded with a ligature. The wound was closed and the animals were placed back in their cages to recover from anesthesia. After $20 \mathrm{~min}$, the mice were treated with $\mathrm{VEH}$ (isotonic saline solution, $7 \mathrm{ml} / \mathrm{kg}$, i.g.) or with ETOH $(7 \mathrm{ml} / \mathrm{kg})$. Four hours later, mice were sacrificed, the abdomen opened, and the stomach was removed after clamping the pylorus and the lower end of the esophagus. By opening the stomach along its greater curvature the contents were collected in a graduated centrifuge tube. The gastric contents were centrifuged at
$3,000 \mathrm{rpm}\left(8,000 \times \mathrm{g}, 25^{\circ} \mathrm{C}\right)$ for $10 \mathrm{~min}$. The gastric $\mathrm{pH}$ values were measured with a $\mathrm{pH}$ meter (HANNA Instruments $\mathrm{pH}$ 211).

2.7. Repeated-Dose 20-Day Toxicity Evaluation of CpMet in Mice. The toxicity of CpMet was assessed in mice according to Lorke's model [24] with modifications. The animals were separated in six different groups $(n=10)$ : one control group (vehicle; isotonic saline solution) and five different treatment groups. For the treatment groups, CpMet was orally administered during 20 consecutive days under one of the two following schemes: (1) CpMet $(10,100$ or $1,000 \mathrm{mg} / \mathrm{kg})$ administration once a day and (2) CpMet (100 or $300 \mathrm{mg} / \mathrm{kg}$ ) administration twice a day. Five animals from each group were sacrificed immediately after the last vehicle or CpMet administration, and another five animals were euthanized 15 days after the last day of administration. Changes in body weight, behavior, and excretions (urinary, fecal, and mucus) were daily recorded and compared with negative controls. Blood samples were obtained by cardiac puncture for the determination of hematological (total erythrocyte count, hemoglobin, hematocrit, total and differential leukocyte count, platelet count, and total protein) and biochemical parameters (glucose, cholesterol, triglycerides, alanine amino transferase, aspartate amino transferase, alkaline phosphatase, bilirubin, urea, creatinine, BUN, and uric acid). Moreover, the mice were dissected and different organs (liver, kidneys, spleen, small bowel, stomach, and large bowel) were excised. Changes in weight and in the macroscopic morphology were recorded. For histopathology examination, the specimens were fixed and processed following standard histological techniques.

2.8. Gastric Ulcer Resolution. The effect of CpMet on the gastric ulcer resolution was evaluated as follows: the gastric ulcer was induced by intragastric instillation of ETOH $(7 \mathrm{ml} / \mathrm{kg})$. After 24 hours of recovery, mice were separated into 4 groups ( $n=20$ per group) and were orally treated for 20 consecutive days under one of the following experimental conditions: (1) VEH (isotonic saline solution, $7 \mathrm{ml} / \mathrm{kg}$ ); (2) carbenoxolone (50 mg/kg), 2 doses per day; (3) CpMet (100 mg/kg), 2 doses per day; and (4) CpMet (300 mg/kg), 2 doses per day. Five mice from each group were sacrificed on the 5th, 10th, 15th, and 20th day after the beginning of the treatment and the gastric ulcer resolution was assessed by macroscopical, histopathological, and histochemical analysis of the stomachs. As it was done for the 20-day toxicity test, changes in body weight, behavior, and excretions (urinary, fecal, and mucus) were daily recorded and compared with negative controls.

The CpMet doses employed for the test were selected based on previous experiments in order to ensure the maximum effect on the resolution of gastric ulcers.

2.9. Histological Analysis. For the ethanol-induced gastric ulcer assessment, the stomachs were dissected out, insufflated with $2 \mathrm{ml}$ of $10 \%$ neutral buffered formalin, and fixed in the same solution until the histological processing. The stomachs were opened throughout the great curvature. The glandular 
portion of the stomach was embedded in paraffin wax and sagittal sections of $5 \mu \mathrm{m}$ of thickness were obtained. Tissue sections were stained with hematoxylin-eosin (H\&E) and periodic acid Schiff (PAS). For the toxicity histopathological examination, the specimens were fixed in $10 \%$ neutral buffered formalin and processed following standard histological techniques and stained with H\&E. Tissue sections were examined under light microscope for morphological or cellular changes.

2.10. Statistical Analysis. Data are presented as mean \pm SEM of $n \geq 6$ per group and at least two independent replicates. Statistically significant differences between the treatments were tested by one-way ANOVA followed by Tukey's test. $P<$ 0.05 was considered statistically significant. GraphPad Prism version 5.00 for Windows, GraphPad Software, La Jolla, California, USA (https://www.graphpad.com), was used for statistics and plotting.

\section{Results}

3.1. Gastroprotective Mechanism of CpMet. To determine the CpMet gastroprotective mechanism, a three-step murine protocol was performed: (1) pretreatment administration (with L-NAME, or INDO, or NEM, or GLIB), (2) CpMet administration $(100 \mathrm{mg} / \mathrm{kg})$, and (3) ETOH administration to induce gastric damage. In order to validate the protocol, each pretreatment was administered in a concentration intended to produce a significant higher damage than ETOH alone. As it can be seen in Figure 1(a), the lesion area induced by the four pretreatments did not show any statistical significant difference among them, but they were higher compared to control.

Figure 1(b) shows the results obtained when CpMet was administered after each pretreatment. Comparing the lesion areas induced by ETOH in the absence or presence of CPMet $(\mathrm{VEH}+\mathrm{VEH}+\mathrm{ETOH}$ versus $\mathrm{VEH}+\mathrm{CpMet}+\mathrm{ETOH})$, it can be observed that the extract reduced by $64.0 \pm 1.56 \%$ the lesion area. Moreover, the four pretreatments with either L-NAME, INDO, NEM, or GLIB (Figure 3(b)) significantly attenuated or abolished the gastroprotective effect of CpMet.

\subsection{Effect of CpMet on the pH of Gastric Contents of Ethanol} Ulcerated Mice. Figure 2(a) shows that the administration of CpMet by itself induced a significant $\mathrm{pH}$ reduction of the gastric contents (final $\mathrm{pH}=3.6)$ compared to $\mathrm{VEH}(\mathrm{pH}=$ 5.4) or omeprazole ( $\mathrm{pH}=6.1$, no significant difference versus control group) treated animals. When the ETOH injury was imposed (Figure 2(b)), the $\mathrm{pH}$ values decreased significantly in the animals treated only with the vehicle. CPMet treated animals exhibited the same behavior as the vehicle. As expected, the treatment with omeprazole clearly reduced acid secretion under injurious conditions (Figure 2(b)).

3.3. CpMet Toxicity Evaluation. The lethality and the toxic adverse effects generated by prolonged CpMet oral administration were assessed considering the conditions of the gastric ulcer resolution assay (Section 3.4.). CpMet toxicity was evaluated in mice using the following treatment schemes

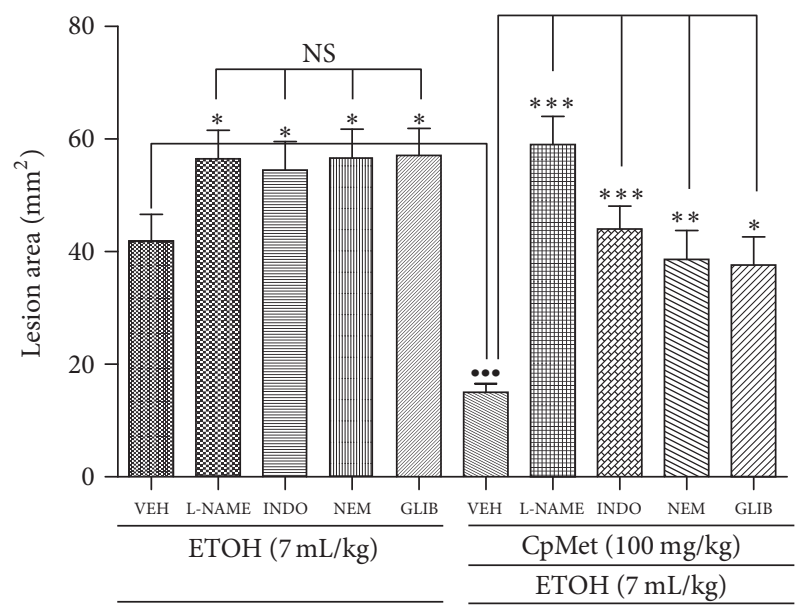

(a)

(b)

FIGURE 1: CpMet gastroprotective mechanism in an ethanol-induced gastric ulcer model in mice. (a) Protocol validation and (b) effect of CpMet. CpMet, C. procera methanolic extract $100 \mathrm{mg} / \mathrm{kg}$; VEH, isotonic saline solution; L-NAME, $\mathrm{N}^{\omega}$-nitro-L-arginine methyl ester $10 \mathrm{mg} / \mathrm{kg}$; INDO, indomethacin $10 \mathrm{mg} / \mathrm{kg}$; NEM, N-ethylmaleimide $1 \mathrm{mg} / \mathrm{kg}$; GLIB, glibenclamide $5 \mathrm{mg} / \mathrm{kg}$. Each column represents the mean \pm SEM of three independent replicates ( $n=6$ per group). An ANOVA was performed followed by Tukey's test. For (a), ${ }^{*} P<$ 0.05 , significant difference versus control (VEH); NS, no significant difference between the four pretreatments. For (b), ${ }^{\cdots} P<0.001$, significant difference $(\mathrm{VEH}+\mathrm{CpMet}+\mathrm{ETOH})$ versus control $(\mathrm{VEH}$ $+\mathrm{VEH}+\mathrm{ETOH}) ;{ }^{*} P<0.05,{ }^{* *} P<0.01,{ }^{* * *} P<0.001$ significant difference between (pretreatment $+\mathrm{CpMet}+\mathrm{ETOH}$ ) versus control $(\mathrm{VEH}+\mathrm{CpMet}+\mathrm{ETOH})$.

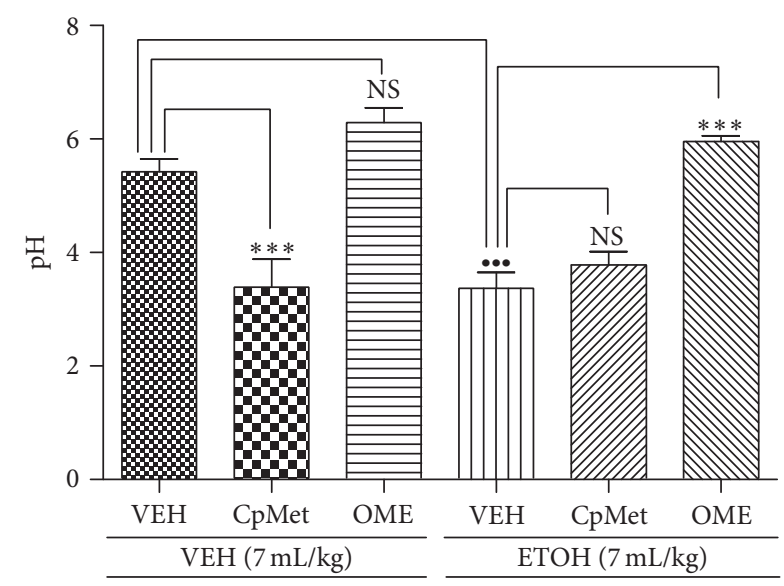

(a)

(b)

Figure 2: Effect of CpMet on gastric pH value. (a) Effect of CpMet and OME on basal gastric pH. (b) Effect of CpMet and OME on the $\mathrm{pH}$ value in $\mathrm{ETOH}$-induced gastric injury. $\mathrm{VEH}$, isotonic saline solution; CpMet, C. procera methanolic extract $100 \mathrm{mg} / \mathrm{kg}$; OME, omeprazole $20 \mathrm{mg} / \mathrm{kg}$. Each column represents the mean \pm SEM of three independent replicates with 6 animals per group. An ANOVA was performed followed by Tukey's test. For (a), ${ }^{* * *} P<$ 0.001 , significant difference versus control (VEH); NS, no significant difference versus control (VEH). For (b), ${ }^{\cdots} P<0.001$, significant difference of $(\mathrm{VEH}+\mathrm{ETOH})$ versus control $(\mathrm{VEH}+\mathrm{VEH}) ;{ }^{* * *} \mathrm{P}<$ 0.001, significant difference versus control (VEH + ETOH); NS, no significant difference versus control $(\mathrm{VEH}+\mathrm{ETOH})$. 


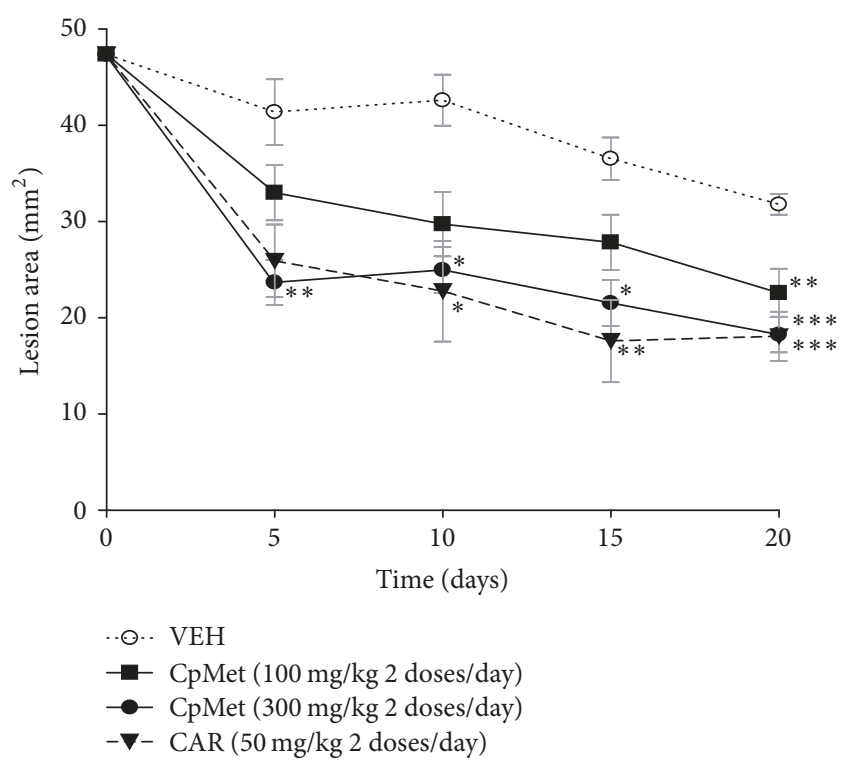

FIGURE 3: Effect of CpMet on the resolution of ethanol-induced gastric ulcers in mice. The graph shows the reduction of the lesion area over time, induced by a continuous 20-day administration of different treatments. VEH, isotonic saline solution; CpMet, C. procera methanolic extract; CAR, carbenoxolone. Each point represents the mean \pm SEM of two independent replicates ( $n=5$ animals). An ANOVA analysis followed by Tukey's test was performed. ${ }^{*} P<0.05$, ${ }^{* *} P<0.01,{ }^{* * *} P<0.001$, significant difference versus control (VEH at the corresponding time).

during a 20-day continuous administration: (I) CpMet (10, 100 , or $1,000 \mathrm{mg} / \mathrm{kg}$ ) once a day and (II) CpMet (100 or $300 \mathrm{mg} / \mathrm{kg}$ ) twice a day. Each group of animals was sacrificed in two stages: half of the group after the last administration (to analyze CpMet direct effects) and the other half, 15 days after the last treatment dose (to check animal recovery in the case that CPMet could produce any alteration).

Under the tested treatment schemes, no lethal effect was observed in any of the experimental animals. The hematological and biochemical parameters analyzed did not show significant alterations in the two sampling stages compared with the control groups (data not shown). Additionally, no significant abnormalities in body weight, behavior, macroscopic organ morphology (including weight), and histopathological appearance were detected (data not shown). Regarding body excretions, only changes in feces consistency (softness) were observed during the initial CpMet administration, but this condition was reversed after 5-7 days of continuous administration. In summary, a 20-day continuous CpMet regimen did not produce any clinical damage, supported by normal histopathological, hematological, hepatic, and renal function tests.

3.4. Effect of CpMet on the Resolution of Ethanol-Induced Gastric Ulcers. Once the toxicological safety of the extract is ascertained, its effect on the resolution of ethanol-induced gastric ulcers was evaluated. Mice were treated with CPMet for a 20-day continuous oral administration of $100 \mathrm{mg} / \mathrm{kg}$ (twice a day) or $300 \mathrm{mg} / \mathrm{kg}$ (twice a day). The effect of the extract on $G U$ resolution was assessed on the 5th, 10th, 15 th, and 20th day by macroscopical, histopathological, and histochemical analysis.

Figure 3 shows a reduction in the lesion area with each one of the treatments over time. As it can be seen, when $\mathrm{VEH}$ was administered to mice, there was a spontaneous ulcer resolution process, reaching on the 20th day a $35 \%$ diminution in the lesion area (from $48.89 \pm 3.05 \mathrm{~mm}^{2}$ to $31.78 \pm 0.78 \mathrm{~mm}^{2}$ ). Regarding the effect of CpMet, on the 20th day of the regimen, a $53.82 \%$ healing effect was observed with $100 \mathrm{mg} / \mathrm{kg}$ (twice a day) compared to the original damage $(t=0)$. When the dose was increased to $300 \mathrm{mg} / \mathrm{kg}$, administered twice a day, the CpMet healing effect became more evident with respect to the previous dose used, affording a significantly $62.65 \%$ reduction in the area of the initial ulcer (from $48.89 \pm 3.05 \mathrm{~mm}^{2}$ to $18.26 \pm 1.45 \mathrm{~mm}^{2}$ ). The positive control CAR, at $50 \mathrm{mg} / \mathrm{kg}$, twice a day, speeded up the healing of gastric ulcer in a similar way compared to the higher dose of CpMet, reducing the original wound area in a $63 \%$.

Comparing the CpMet healing effect with the VEH, the $300 \mathrm{mg} / \mathrm{kg}$ dose, was the only treatment with statistical significance at all the sampled times $(P<0.05$ to 0.001$)$. The CAR treatment (positive control) was also significantly different compared to the $\mathrm{VEH}$, except for the result recorded on the 5 th day of sampling (Figure 3 ).

If we consider the remaining damage obtained at the 20th day with the $\mathrm{VEH}$ treatment (lesion area $=31.78 \pm 0.78$ ) as the $100 \%$, we can calculate a net healing value of $42.54 \%$ with CpMet $300 \mathrm{mg} / \mathrm{kg}$, twice a day. Performing the same calculation, a twice a day administration with $50 \mathrm{mg} / \mathrm{kg}$ of CAR, resulted in a $43.17 \%$ net healing.

3.4.1. Macroscopic Examination of Stomachs. Figure 4(a) shows a representative macroscopic image of a normal stomach with a smooth surface and no visible scars. In Figures 5(a), 6(a), and 7(a), representative stomach images, on the 20th day of the experiment, of controls and CpMet $(300 \mathrm{mg} / \mathrm{kg}$, twice a day) treated mice are depicted. Figure 5(a) shows a stomach of the negative control group (VEH) where many ethanol-induced scars are visible. It has to be noticed that, after 20 days of the ulcer induction with ethanol, the lesions observed are usually characterized by the presence of rounded scars without the hemorrhage or edema of an acute ulcer. Figure 6(a) presents a CAR $(50 \mathrm{mg} / \mathrm{kg}$, twice a day) positive control stomach, where a substantially improved appearance of the gastric surface compared to VEH (Figure 5(a)) is observed. The stomach is smoother and only a few small rounded scars are visible. Finally, in Figure 7(a), the stomach of the CpMet $(300 \mathrm{mg} / \mathrm{kg}$, twice a day) treated animals exhibits similar healing characteristics as the positive control (Figure 6(a)). This observation is supported by the statistical analysis where both treatments (CAR and CPMet) reached the same significance level (Figure 3).

3.4.2. Histochemical and Histopathological Analysis. To ascertain the previous macroscopic observations, a histopathological analysis was performed. The histological inspection of 


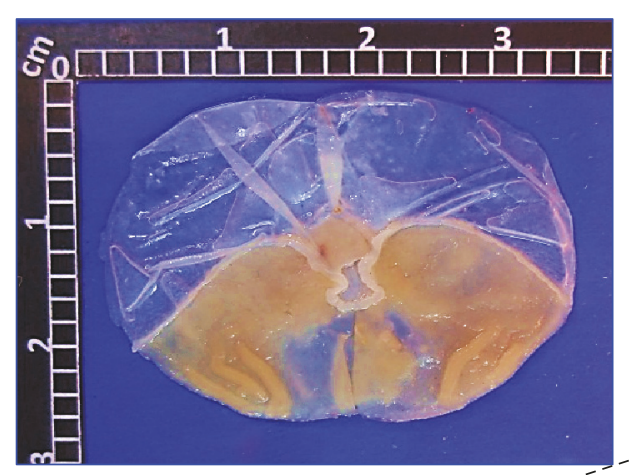

(a)

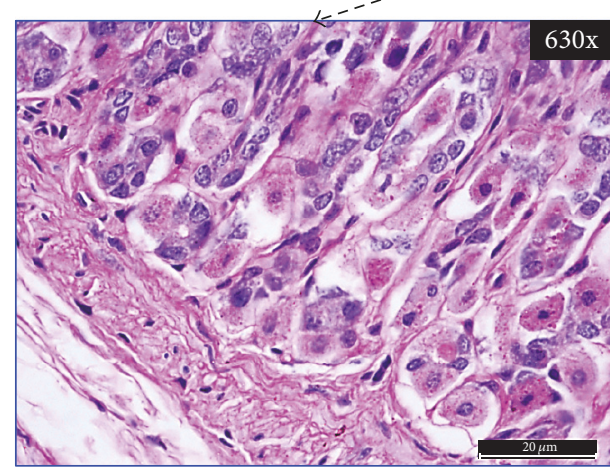

(c)

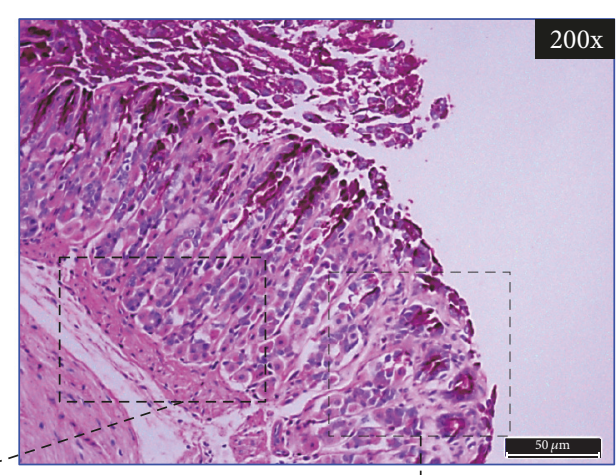

(b)

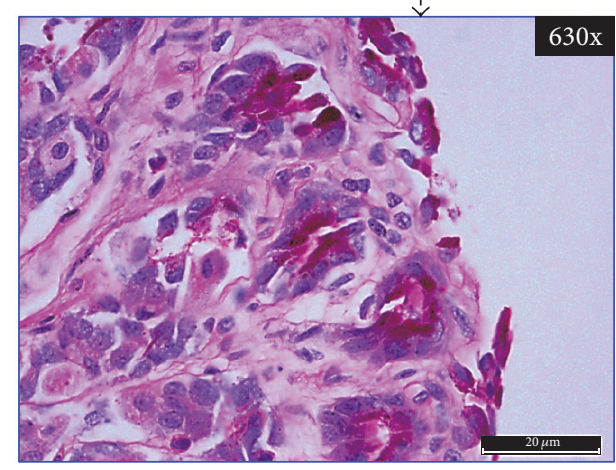

(d)

FIgURE 4: Normal control. Mice stomach appearance without any treatment. (a) Macroscopic view of a fresh dissected stomach without injuries. (b), (c), and (d) Bright-field microscopic views of $5 \mu \mathrm{m}$ thickness sagittal sections of the glandular portion of the stomach, stained with PAS. (b) Panoramic histochemical appearance showing tissue architectural integrity. Fuchsia clusters indicate PAS positivity and the presence of neutral mucus surrounding the mucosal epithelium. Dashed boxes: right, magnification to show mucus presence; left, histological status of the tissue. (c) Histological observation of the gastric mucosa showing well-organized mucosa structures without basal inflammatory infiltrate. (d) Histochemical analysis of the normal gastric mucosa of mice displaying PAS-positive neutral mucosubstances that predominate in the entire gastric surface.

a normal gastric mucosa exhibits the mucosal surface and the gastric pits lined by columnar epithelial cells as well as PAS-stained neutral mucins and no inflammatory cell infiltrate, indicating an intact gastric mucosa layer (Figures 4(b), 4(c), and 4(d)). Figures 5(b) and 5(d), corresponding to VEH treated mice on the 20th day of the experiment, show a significantly reduced PAS-positive adherent gastric mucus in the surface cells compared with normal mice (Figures 4(b) and 4(d)). Furthermore, diffusive erosion of the gastric mucosal cell layer with distorted and fragmented cells besides an increased amount of inflammatory cells throughout the mucosa and submucosa is observed (Figures $5(\mathrm{~b})$ and 5(c)). In contrast, on the 20th day, the apical surface of the gastric mucous cells in CAR $(50 \mathrm{mg} / \mathrm{kg}$, twice a day) treated mice reveals a strong PAS-positive staining and a lack of inflammatory cells (Figures 6(b), 6(c), and 6(d)). On the other hand, compared with the control group (VEH), CpMet $(300 \mathrm{mg} / \mathrm{kg}$ twice a day) treated mice exhibit an alleviation of the erosions in the gastric mucosal cell layer and an increased PAS reaction, indicating a higher production of mucosubstances (Figures 7(b) and 7(d)). In addition to the remarkable mucus production, no inflammatory (neutrophil/monocyte) infiltration of the mucosa is observed (Figures 7(b) and 7(c)). An almost similar pattern, without inflammatory infiltration and augmented mucus production evidenced by PASpositive clusters lining the mucosal surface, is observed in the photomicrographs of CpMet $100 \mathrm{mg} / \mathrm{kg}$, twice a day treated mice (data not shown). Nevertheless, the healing effect of this dose is not enough to alleviate the GU in a 20-day period as it could be perceived in the macroscopic images as well as by the presence of a slightly disrupted regeneration of the apical surface (data not shown).

\section{Discussion}

In a previous work, we reported the remarkable gastroprotective activity of $C$. procera bark methanolic extract (CpMet), as well as its anti- $H$. pylori activity [21]. On this basis, the present study was performed to examine the mechanisms by which $C p M e t$ prevents gastric damage and to determine if a continued CpMet treatment promotes the resolution of preexisting gastric ulcers.

Considering that gastric ulcers develop as a result of an imbalance between defensive and injurious gastric mucosal 


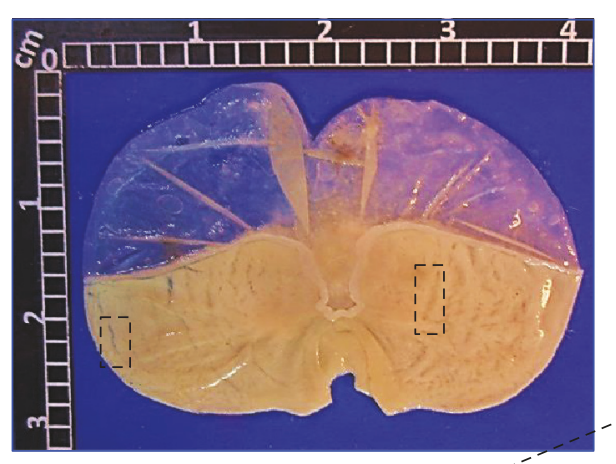

(a) - - -

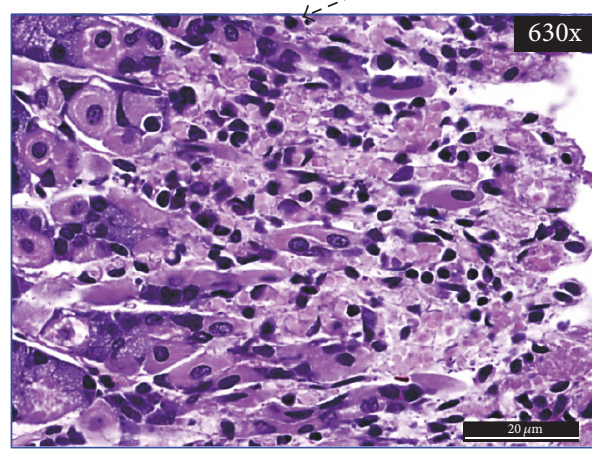

(c)

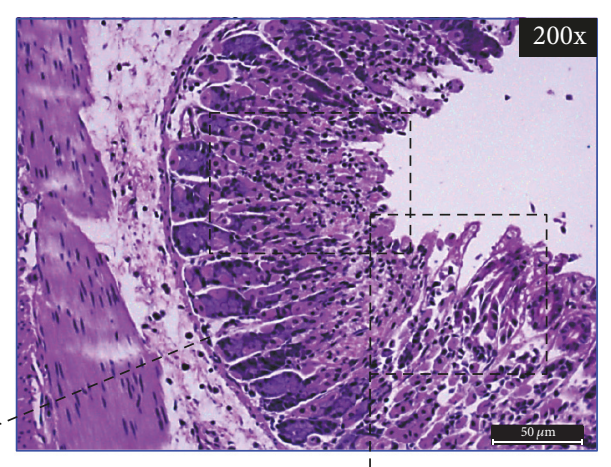

(b)

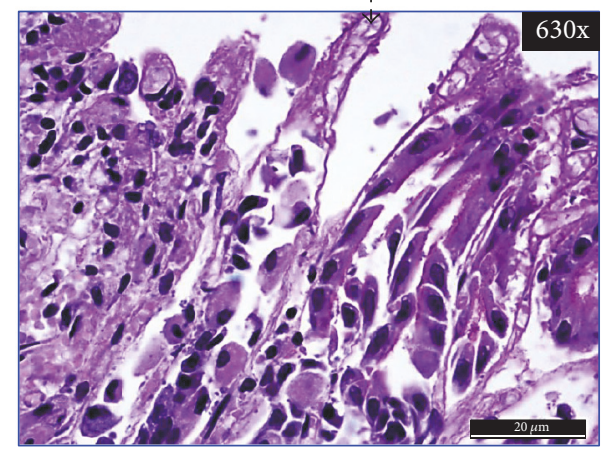

(d)

FIGURE 5: Negative control. Mice stomach treated with isotonic saline solution ( $7 \mathrm{ml} / \mathrm{kg}$ twice a day) on the 20th day after GU induction. (a) Macroscopic view of a formalin fixed stomach. Dashed boxes show the typical scars observed after 20 days of the GU induction with ethanol. (b), (c), and (d) Bright-field microscopic views of $5 \mu \mathrm{m}$ thickness sagittal sections of the glandular portion of the stomach, stained with PAS. (b) Panoramic histopathological appearance showing an extended degree of mucosal damage with disorganized architecture and basophilic staining due to persistent leukocyte infiltration. Dashed boxes: right, magnification to show mucus presence; left, histopathological status of the tissue. (c) Histopathological observation of the gastric mucosa revealing diffusive erosion of the gastric mucosa cell layer and abundant inflammatory cells present in the lamina propria. (d) Histochemical analysis presenting weak PAS positivity due to loss of epithelial continuity in the mucosa surface and gastric mucous neck cells.

factors, it was needed to assess the effect of CpMet on the gastric protective mechanisms.

There are several molecules that specifically block some of the endogenous gastroprotective mechanisms. Among the most studied and best characterized are L-NAME, INDO, NEM, and GLIB, involved with endogenous NO synthesis, prostaglandins (PGs) production, sulfhydryl groups, and ATP-sensitive potassium $\left(\mathrm{K}_{\mathrm{ATP}}\right)$ channels, respectively. As expected, the pretreatment with L-NAME, or INDO, or NEM, or GLIB effectively suppressed the targeted gastroprotective mechanism (Figure 1(a)). The administration of CpMet before the ethanol-induced injury clearly produced a remarkable gastroprotective activity $(64.0 \pm 1.56 \%$ reduction of the lesion area). Nevertheless, when the extract was administered after the four pretreatments, the antiulcer effect of CpMet was abolished or attenuated (Figure 1(b)), indicating that the extract gastroprotective activity is related to the four mechanisms evaluated. However, a multiple means comparison among the treatments showed a greater dependence on endogenous NO and PGs, followed by sulfhydryl groups and $\mathrm{K}_{\text {ATP }}$ channels.
Intragastric administration of absolute ETOH to experimental animals is a widely used model to induce gastric ulceration. The pathogenesis of ethanol-induced gastric ulcers is complex and provokes a broad spectrum of metabolic and functional changes, which include an increase in acid secretion, oxidative stress, and depletion of nonprotein sulfhydryl (NP-SH) compounds, diminishment of the gastric mucosal blood flow, impairment of the NO pathway, and decreased PGs synthesis [25-28].

NO has been recognized as an important mediator in the maintenance of gastric mucosal integrity [29], through the modulation of gastric mucosal blood flow, mucus, and bicarbonate secretions [30]. Thus, the impairment in NO synthesis affects the gastric microcirculation [25]. In the present work, the inhibition of NO synthesis with L-NAME clearly increased the lesion area induced by ETOH administration. In the group receiving $C p M e t$ with the $\mathrm{NO}$-synthase inhibitor, the lesion area remained the same as in the untreated group (without the extract) (Figure 1). This data suggests that the maintenance of NO production is crucial for the gastroprotective mechanism of CpMet, perhaps by preventing gastric microcirculation damage. 


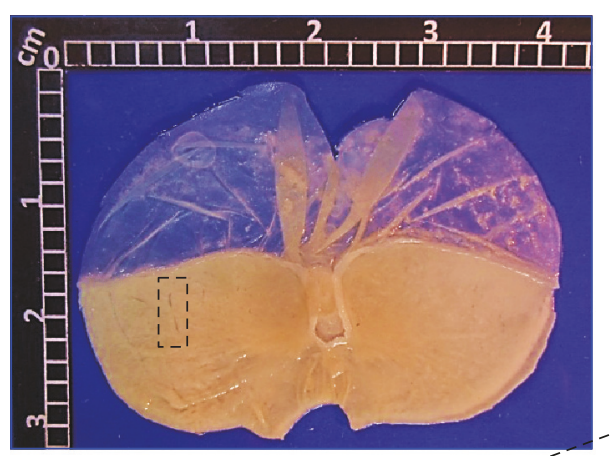

(a) - - -

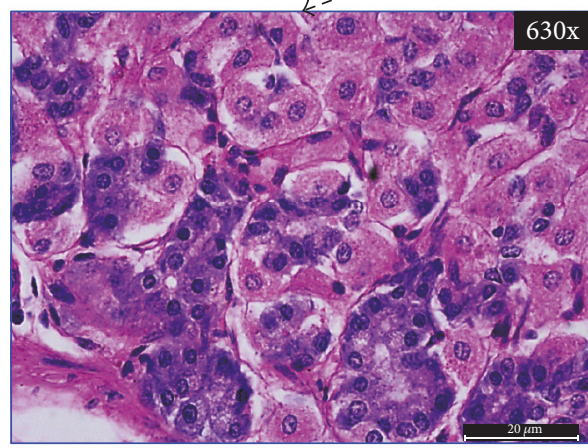

(c)

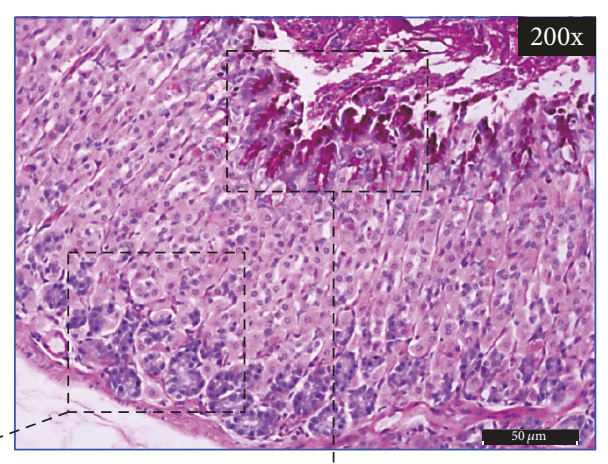

(b)

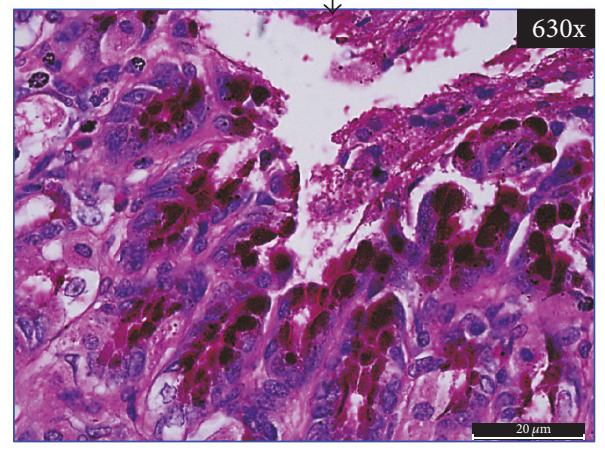

(d)

Figure 6: Positive control. Mice stomach treated with CAR $(50 \mathrm{mg} / \mathrm{kg}$, twice a day) on the 20th day after GU induction. (a) Macroscopic view of a formalin fixed stomach showing only some minor scars (dashed box). (b), (c), and (d) Bright-field microscopic views of $5 \mu \mathrm{m}$ thickness sagittal sections of the glandular portion of the stomach, stained with PAS. (b) Panoramic histopathological appearance showing robust basal architecture with some alterations at the apical portion of the mucosa and abundant clusters of PAS-positive staining regions. Dashed boxes: right, magnification to show mucus presence; left, histopathological status of the tissue. (c) Gastric mucosa revealing normal glandular organization without inflammatory infiltrate. (d) Gastric mucosa with strong PAS positivity surrounding the epithelia and areas of expanded surface mucous cells. In some areas, a slightly disrupted regeneration of the gastric epithelium is observed.

PGs serve as gastric cytoprotectants by attenuating or preventing mucosal lesions. In fact, the inhibition of their synthesis by blocking the cyclooxygenase enzyme with NSAIDs such as indomethacin results in the reduction of gastric mucosal blood flow and mucosal damage. In the present study, the gastroprotection favored by CpMet was attenuated by INDO pretreatment. The same behavior was attained when blocking $\mathrm{K}_{\mathrm{ATP}}$ channels with GLIB. The protective effect exerted by PGs is, at least in part, mediated by the activation of $\mathrm{K}_{\mathrm{ATP}}$ channels [31], contributing to improved gastric microcirculation. Regarding that the lesion area generated in the presence of CpMet is sensitive to both INDO and GLIB, it is likely that the partial gastric protection afforded may be related to maintaining a moderate production of PGs, which in turn, act as $\mathrm{K}_{\mathrm{ATP}}$ channel activators.

NP-SH compounds protect gastric mucosa by controlling mucus production and binding free radicals. Previous reports have shown that NP-SH compounds are depleted in ETOHinduced gastric lesions [32]. In this work, the ulcerogenic effect mediated by the administration of NEM and ETOH was partially reduced by CpMet treatment, suggesting that, in the gastroprotective mechanism of the extract, probably underlies the elimination of the noxious free radicals through the participation of endogenous NP-SH.

Regarding the effect of CpMet on gastric $\mathrm{pH}$ (Figure 2), our results suggest that the extract did not have a favorable effect on this parameter; however it is noteworthy to mention that the administration of the extract did not intensify the acidity of gastric contents induced by ETOH alone, probably due to a local effect of CpMet that prevents ETOH absorption and its outcomes on gastric $\mathrm{pH}$. The ulcerogenic effect of ETOH is based on an increase in the secretion of histamine, pepsin, and $\mathrm{H}^{+}$ions, among other deleterious elements; nevertheless, ethanol-induced gastric ulcers are not inhibited by antisecretory drugs such as cimetidine [33]. In this sense, it appears to be more important to improve the mucosal defensive mechanisms rather than just blocking the acid production. On the basis of our data, it can be suggested that the gastroprotective effect of CpMet does not rely on the gastric acid inhibition; however, it cannot be ruled out its antiulcer potential even in the absence of an acid antisecretory effect, since the extract could serve as a coadjuvant of conventional antiacid treatments. Moreover, the absence of an antiacid mechanism of CpMet encourages its beneficial protective 


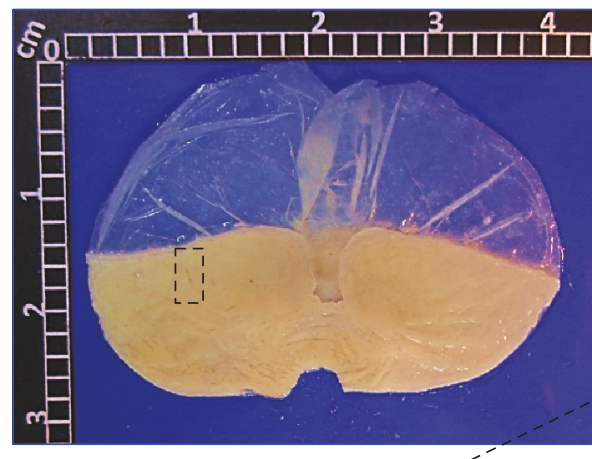

(a) - -

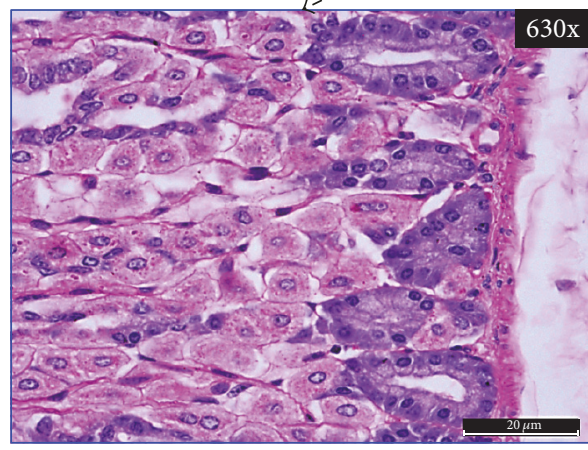

(c)

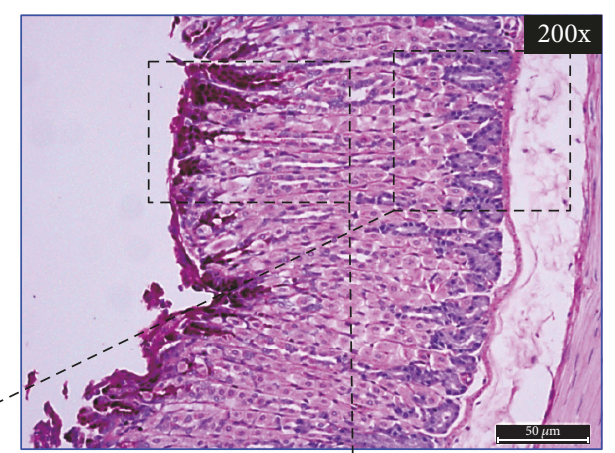

(b)

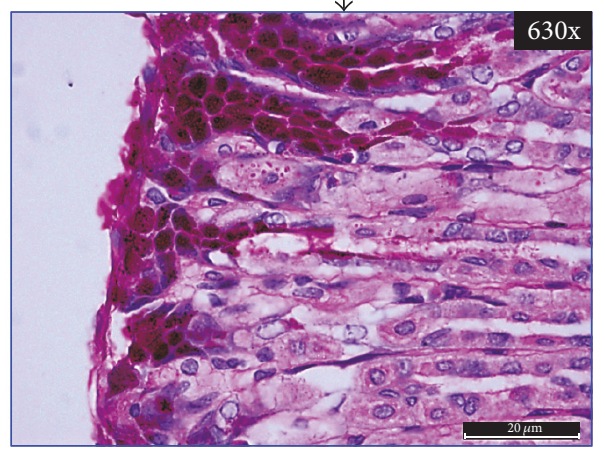

(d)

Figure 7: CpMet treatment. Mice stomach treated with CpMet $(300 \mathrm{mg} / \mathrm{kg}$ twice a day) on the 20th day after GU induction. (a) Macroscopic view of a formalin fixed stomach mainly showing a smooth surface with few flat small scars (dashed box). (b), (c), and (d) Brightfield microscopic views of $5 \mu \mathrm{m}$ thickness sagittal sections of the glandular portion of the stomach, stained with PAS. (b) Panoramic histopathological appearance revealing a good organization of the basal gastric mucosa architecture with only minor alterations at the apical portion of the mucosa and abundant PAS-positive clusters lining the epithelium. Dashed boxes: left, magnification to show mucus presence; right, histopathological status of the tissue. (c) Histopathological observation displaying well-organized mucosal architecture, no inflammatory infiltrates are observed. (d) Gastric mucosa with strong PAS positivity surrounding the epithelium and areas of expanded mucous neck cells secreting increased amounts of mucins. Normal and well-defined mucous producing cells are observed.

effects, taking into account the fact that long-term therapies based on modifications of acid secretion are related to adverse side effects (e.g., hypergastrinemia) due to augmented $\mathrm{pH}$ in the gastric lumen [33].

Ethanol administration induces gastric ulcers that spread over large areas: lesions that are characterized by persistent leakage and increased blood flow stasis at the ulcer margin accompanied by edema, congestion of the surface epithelium, and inflammatory infiltration. The assessment of CpMet effect on the resolution of gastric ulcers induced by ethanol revealed that a 20-day continuous oral administration of the extract at dose of $300 \mathrm{mg} / \mathrm{kg}$, twice a day, clearly reduced the lesion area (62.65\%) (Figure 3); it has to be pointed out that the main effect relied on the time required to significantly reduce the initial lesion area, which was only about 5 days. Thus, comparing the ulcer resolution obtained on the 5 th day with CpMet $300 \mathrm{mg} / \mathrm{kg}$ (twice a day) and the negative control (VEH), a $42.73 \%$ of improvement was attained, while $20.23 \%$ of healing was reached with $100 \mathrm{mg} / \mathrm{kg}, 2$ doses per day. This data shows a dose-dependent effect and indicates the necessity of a high and reinforced CpMet dose $(300 \mathrm{mg} / \mathrm{kg}$, twice a day) to get a significant early gastric resolution. It has to be emphasized that this latter dose of CpMet administered twice a day is the one that ensured the maximum healing effect, since previous dose-response experiments (data not shown) revealed that single doses per day of the extract were not enough to obtain the resolution effect. Further studies are required to elucidate the pharmacokinetics and pharmacodynamics of CpMet, which may explain the necessity of a twice daily dose, besides being a critical requirement for developing a safe and effective phytomedicine.

Numerous studies have shown that current antiulcer drugs, targeted to inhibit acid secretion, are insufficient to promote a complete ulcer healing and to prevent the relapse of gastric ulcers. The resolution of a gastric ulcer involves the formation of a scar through migration and proliferation of epithelial and connective tissue cells, besides active angiogenesis and extracellular matrix deposition [13]. It has been suggested that the quality with which this repair process occurs plays a key role in the risk of ulcer recurrence. On this basis, the concept of quality of ulcer healing (QOUH) based on the histological maturity of regenerated mucosa of healed ulcers has been proposed. PGs and some growth factors (e.g., VEGF) have been positively associated with an 
improved QOUH, whereas the persistence of an increased infiltration with neutrophils and macrophages results in an immature regenerated area with distorted architecture and prone to ulcer recurrence [11].

When an external aggression, such as ethanol, alters the gastric mucosa homeostasis, gross histological changes linked to the ulcerative process can be observed. Although, gastric mucosa activates different responses to alleviate damage, after 20 days of $\mathrm{GU}$ induction, the mucosal recovery is not fully completed. In the context of the present work, the results show that CpMet $(300 \mathrm{mg} / \mathrm{kg}$, twice a day) has a strong cicatrisation activity by reaching a net healing value of $42.54 \%$ (on the 20th day), a percentage that is even afforded since the 5 th day of treatment (Figure 3). Moreover, unlike VEH treatment, the stomachs of CpMet (300 mg/kg, twice a day) treated mice exhibited flat scars with an increased mucus production and most importantly, without inflammatory infiltration, which seems to be the basis of a good QOUH. In essence, the flat macroscopical appearance of the scars generated with CpMet treatment dovetails with the histochemical findings, by showing a marked improvement in the gastric ulcer resolution. Further research should be done to clarify the precise mechanism by which the extract exerts its effect on the resolution of gastric ulcers; however, in the light of our results, it seems that CpMet has a potent anti-inflammatory effect by limiting the attraction and accumulation of inflammatory cells.

There are many works that have sought the cure of gastric ulcers by testing compounds derived from plants; however, herbal extracts have shown to be a better treatment option, or an excellent choice as adjuvants, to prevent or promote an effective healing effect due to the multiple activities they can exert simultaneously (i.e., antibiotic, antiulcerogenic, antiinflammatory, antioxidant, angiogenic, and cytoprotective) $[12,13]$. Referring specifically to extracts or preparations from plants, there are several studies that have assessed their gastroprotective and ulcer healing activities. Indeed, many of these works have demonstrated that the efficacy to prevent or ameliorate gastric ulcers is comparable or even better than that of some drugs used in conventional therapies (i.e., sucralfate, omeprazole, or cimetidine). However, the beneficial effects vary extensively depending on the injurious agent used to induce the ulceration (NSAID's, ethanol, acetic acid, cold water restraint stress, $\mathrm{HCl}$, or pyloric ligation), the plant species, type of extract, and the treatment duration. For instance, in a review performed by $\mathrm{Bi}$ et al. [12], the percentage of efficacy in treating gastric ulcers with only one or a combination of herbal extracts ranged from 20 to $\sim 80 \%$. In another study performed by Mota da Silva et al. [34], an hydroalcoholic extract of Maytenus robusta reduced the gastric ulcer area by $53 \%$, and the healing effectiveness seemed to be mediated by increased gastric mucin content and reduced oxidative stress and inflammatory parameters at the site of the ulcer; nevertheless, it seems that the extract did not reduce the leukocyte migration as in the case of CPMet.

Although it is true that there are a vast number of studies in the literature reporting that diverse herbal extracts promote a significant reduction of gastric ulcers, some of the results are mainly based on the macroscopic measurement of the ulcerated area, leaving aside the histological assessment, which is the cornerstone to ascertaining a good QOUH. Furthermore, it is noteworthy that only a few herbal extracts have available information related to their toxicological safety, which is essential to promote the safe use of herbal medicines.

According to the data obtained in the present study and our previous report [21], CpMet exerts polypharmacological activities, demonstrated by the following findings: (1) in vitro anti- $H$. pylori activity; (2) gastroprotective and ulcer healing effects; and (3) anti-inflammatory action. Additionally, the extract turned out to be safe under subacute administration which, besides its beneficial properties, makes it an attractive candidate to continue its study in preclinical tests. The advantage that CpMet has polypharmacological effects is that it can impact on various known etiological factors of gastric ulcers as well as in the mechanisms that underlie the ulcer resolution. The CpMet anti-H. pylori activity is of great importance considering that this bacterium is responsible for $70-85 \%$ of gastric ulcers [35]. Moreover, the benefits of the extract are related, in a greater or lesser extent, to NO synthesis, PGs production, sulfhydryl groups, and $\mathrm{K}_{\mathrm{ATP}}$ channels, mechanisms that, in general terms, modulate the gastric defenses such as microcirculation, mucus, and ROS production. The processes that mediate the protection of the gastric mucosa are neither independent nor different from the ones that participate in the resolution of gastric ulcers. In this sense, the antiulcer and healing effects of CpMet may rely on an increased mucus production, an anti-inflammatory action, and a partial dependence on PGs, compounds that promote a high QOUH.

\section{Conclusion}

The data obtained in the present work highlights the effectiveness of the methanolic extract of $C$. procera for improving the quality of resolution of a preexisting ulcer by different mechanisms, but mostly through cytoprotective and antiinflammatory effects. Even though conventional treatments are effective in the management of gastric ulceration, they are not sufficient to avoid ulcer recurrences. Thus, CpMet could be a good adjuvant of current therapies, based on its antimicrobial, antiulcer, and gastric healing effects.

\section{Abbreviations}

CAR: Carbenoxolone

CpMet: Cyrtocarpa procera methanolic extract

ETOH: Absolute ethanol

GLIB: Glibenclamide

GU: $\quad$ Gastric ulcers

H\&E: Hematoxylin-eosin

INDO: Indomethacin

$\mathrm{K}_{\mathrm{ATP}}: \quad$ ATP-sensitive potassium

L-NAME: $\mathrm{N}^{\omega}$-Nitro-L-arginine methyl ester

NEM: $\quad$ N-Ethylmaleimide

NO: $\quad$ Nitric oxide

NP-SH: Nonprotein sulfhydryl

NSAIDs: Nonsteroidal anti-inflammatory drugs

OME: Omeprazole

PAS: $\quad$ Periodic acid Schiff

PGs: Prostaglandins 
QOUH: Quality of ulcer healing

ROS: Reactive oxygen species

VEH: Vehicle.

\section{Conflicts of Interest}

The authors declare that there are no conflicts of interest regarding the publication of this article.

\section{Acknowledgments}

This study was partially supported by DGAPA-UNAM (PAPIIT IN216414 and IN214317). The authors thank Mtra. Rosa María Fonseca, Facultad de Ciencias, UNAM, for her support in the collection and identification of C. procera.

\section{References}

[1] J. B. Gibson, S. W. Behrman, T. C. Fabian, and L. G. Britt, "Gastric outlet obstruction resulting from peptic ulcer disease requiring surgical intervention is infrequently associated with Helicobacter pylori infection," Journal of the American College of Surgeons, vol. 191, no. 1, pp. 32-37, 2000.

[2] J.-Y. Lau, J. Sung, C. Hill, C. Henderson, C. W. Howden, and D. C. Metz, "Systematic review of the epidemiology of complicated peptic ulcer disease: incidence, recurrence, risk factors and mortality," Digestion, vol. 84, no. 2, pp. 102-113, 2011.

[3] P. Malfertheiner, F. K. Chan, and K. E. McColl, "Peptic ulcer disease," The Lancet, vol. 374, no. 9699, pp. 1449-1461, 2009.

[4] A. Mejia and W. K. Kraft, "Acid peptic diseases: pharmacological approach to treatment," Expert Review of Clinical Pharmacology, vol. 2, no. 3, pp. 295-314, 2009.

[5] J. J. Y. Sung, E. J. Kuipers, and H. B. El-Serag, "Systematic review: the global incidence and prevalence of peptic ulcer disease," Alimentary Pharmacology \& Therapeutics, vol. 29, no. 9, pp. 938946, 2009.

[6] R. Dimaline and A. Varro, "Attack and defence in the gastric epithelium-a delicate balance," Experimental Physiology, vol. 92, no. 4, pp. 591-601, 2007.

[7] M. I. Gomes Silva and F. C. Florenço de Sousa, "Gastric Ulcer Etiology," in Peptic Ulcer Disease, J. Chai, Ed., InTech, 2011.

[8] M. Magierowski, K. Magierowska, S. Kwiecien, and T. Brzozowski, "Gaseous mediators nitric oxide and hydrogen sulfide in the mechanism of gastrointestinal integrity, protection and ulcer healing," Molecules, vol. 20, no. 5, pp. 9099-9123, 2015.

[9] C. Scarpignato, I. Pelosini, and F. Di Mario, "Acid suppression therapy: where do we go from here?" Digestive Diseases, vol. 24, no. 1-2, pp. 11-46, 2006.

[10] E. Sheen and G. Triadafilopoulos, "Adverse effects of long-term proton pump inhibitor therapy," Digestive Diseases and Sciences, vol. 56, no. 4, pp. 931-950, 2011.

[11] T. Arakawa, T. Watanabe, T. Tanigawa, K. Tominaga, Y. Fujiwara, and K. Morimoto, "Quality of ulcer healing in gastrointestinal tract: its pathophysiology and clinical relevance," World Journal of Gastroenterology, vol. 18, no. 35, pp. 4811-4822, 2012.

[12] W.-P. Bi, H.-B. Man, and M.-Q. Man, "Efficacy and safety of herbal medicines in treating gastric ulcer: a review," World Journal of Gastroenterology, vol. 20, no. 45, pp. 17020-17028, 2014.
[13] N. Kangwan, J. M. Park, E. H. Kim, and K. B. Hahm, "Quality of healing of gastric ulcers: natural products beyond acid suppression," World Journal of Gastrointestinal Pathophysiology, vol. 5, no. 1, pp. 40-47, 2014.

[14] V. Argueta, J. Cano, and M. Rodarte, "Atlas de las plantas de la medicina tradicional mexicana," Instituto Nacional Indigenista, México, vol. I, 1994.

[15] M. Canales, T. Hernández, J. Caballero et al., "Informant consensus factor and antibacterial activity of the medicinal plants used by the people of San Rafael Coxcatlán, Puebla, México," Journal of Ethnopharmacology, vol. 97, no. 3, pp. 429439, 2005.

[16] M. Canales Martínez, T. Hernández Delgado, J. Caballero Nieto, A. Romo de Vivar Romo, Á. Durán Díaz, and R. Lira Saade, "Análisis cuantitativo del conocimiento tradicional de las plantas medicinales en San Rafael, Coxcatlán, valle de tehuacáncuicatlán, Puebla, México," Acta Botánica Mexicana, no. 75, p. 21, 2006.

[17] P. Hersch-Martínez, "Commercialization of wild medicinal plants from southwest Puebla, Mexico," Economic Botany, vol. 49, no. 2, pp. 197-206, 1995.

[18] V. Rodríguez-López, F. Aguirre-Crespo, L. Salazar, and S. Estrada-Soto, "Identification of fatty acid esters and hydrocarbon derivatives from Cyrtocarpa procera Kunth by GC-MS," Natural Product Research (Formerly Natural Product Letters), vol. 20, no. 1, pp. 1-7, 2006.

[19] H. Rosas-Acevedo, T. Terrazas, M. E. González-Trujano, Y. Guzmán, and M. Soto-Hernández, "Anti-ulcer activity of Cyrtocarpa procera analogous to that of Amphipterygium adstringens, both assayed on the experimental gastric injury in rats," Journal of Ethnopharmacology, vol. 134, no. 1, pp. 67-73, 2011.

[20] I. Castillo-Juárez, V. González, H. Jaime-Aguilar et al., "AntiHelicobacter pylori activity of plants used in mexican traditional medicine for gastrointestinal disorders," Journal of Ethnopharmacology, vol. 122, no. 2, pp. 402-405, 2009.

[21] W. I. Escobedo-Hinojosa, J. D. Del Carpio, J. F. PalaciosEspinosa, and I. Romero, "Contribution to the ethnopharmacological and anti-Helicobacter pylori knowledge of Cyrtocarpa procera Kunth (Anacardiaceae)," Journal of Ethnopharmacology, vol. 143, no. 1, pp. 363-371, 2012.

[22] A. Bucciarelli and M. I. Skliar, "Medicinal plants from Argentina with gastro protective activity," Ars Pharmaceutica, vol. 48, no. 4, pp. 361-369, 2007.

[23] H. Shay, S. A. Komarov, S. S. Fels, D. Meranze, M. Gruenstein, and H. Siplet, "A simple method for the uniform production of gastric ulceration in the rat," Gastroenterology, vol. 5, no. 1, pp. 43-61, 1945.

[24] D. Lorke, "A new approach to practical acute toxicity testing," Archives of Toxicology, vol. 54, no. 4, pp. 275-287, 1983.

[25] O. M. E. Abdel-Salam, J. Czimmer, A. Debreceni, J. Szolcsányi, and G. Mózsik, "Gastric mucosal integrity: gastric mucosal blood flow and microcirculation. An overview," Journal of Physiology-Paris, vol. 95, no. 1-6, pp. 105-127, 2001.

[26] A. Bhattacharyya, R. Chattopadhyay, S. Mitra, and S. E. Crowe, "Oxidative stress: an essential factor in the pathogenesis of gastrointestinal mucosal diseases," Physiological Reviews, vol. 94, no. 2, pp. 329-354, 2014.

[27] S. Chari, S. Teyssen, and M. V. Singer, "Alcohol and gastric acid secretion in humans," Gut, vol. 34, no. 6, pp. 843-847, 1993.

[28] A. Szlachcic, G. Krzysiek-Maczka, R. Pajdo et al., "The impact of asymmetric dimethylarginine (ADAMA), the endogenous 
nitric oxide (NO) synthase inhibitor, to the pathogenesis of gastric mucosal damage," Current Pharmaceutical Design, vol. 19, no. 1, pp. 90-97, 2013.

[29] L. Wang, Y. Zhou, J. Peng, Z. Zhang, D.-J. Jiang, and Y.J. Li, "Role of endogenous nitric oxide synthase inhibitor in gastric mucosal injury," Canadian Journal of Physiology and Pharmacology, vol. 86, no. 3, pp. 97-104, 2008.

[30] S. Kwiecien, M. W. Pawlik, T. Brzozowski et al., "Nitric oxide (NO)-releasing aspirin and (NO) donors in protection of gastric mucosa against stress," Journal of Physiology and Pharmacology, vol. 59, pp. 103-115, 2008.

[31] B. M. Peskar, K. Ehrlich, and B. A. Peskar, "Role of ATP-sensitive potassium channels in prostaglandin-mediated gastroprotection in the rat," The Journal of Pharmacology and Experimental Therapeutics, vol. 301, no. 3, pp. 969-974, 2002.

[32] A. L. Rozza, T. de Mello Moraes, H. Kushima, D. S. Nunes, C. A. Hiruma-Lima, and C. H. Pellizzon, "Involvement of glutathione, sulfhydryl compounds, nitric oxide, vasoactive intestinal peptide, and heat-shock protein-70 in the gastroprotective mechanism of Croton cajucara Benth. (Euphorbiaceae) essential oil," Journal of Medicinal Food, vol. 14, no. 9, pp. 10111017, 2011.

[33] C. Takayama, F. M. De-Faria, A. C. A. De Almeida et al., "Gastroprotective and ulcer healing effects of essential oil from Hyptis spicigera Lam. (Lamiaceae)," Journal of Ethnopharmacology, vol. 135, no. 1, pp. 147-155, 2011.

[34] L. Mota da Silva, T. Boeing, L. B. Somensi et al., "Evidence of gastric ulcer healing activity of Maytenus robusta Reissek: in vitro and in vivo studies," Journal of Ethnopharmacology, vol. 175, pp. 75-85, 2015.

[35] T. L. Testerman and J. Morris, "Beyond the stomach: an updated view of Helicobacter pylori pathogenesis, diagnosis, and treatment," World Journal of Gastroenterology, vol. 20, no. 36, pp. 12781-12808, 2014. 


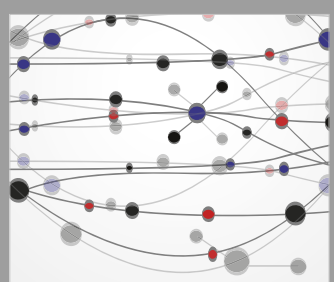

The Scientific World Journal
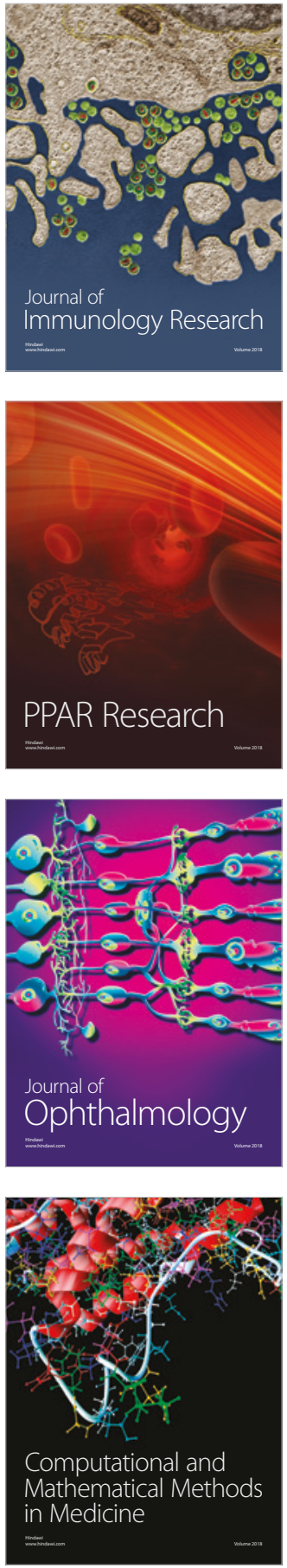

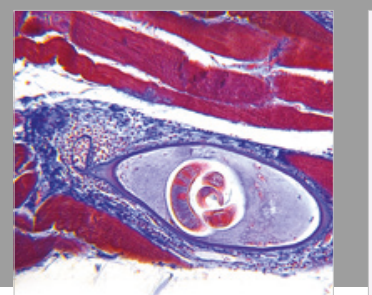

Gastroenterology Research and Practice

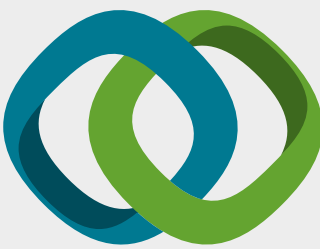

\section{Hindawi}

Submit your manuscripts at

www.hindawi.com
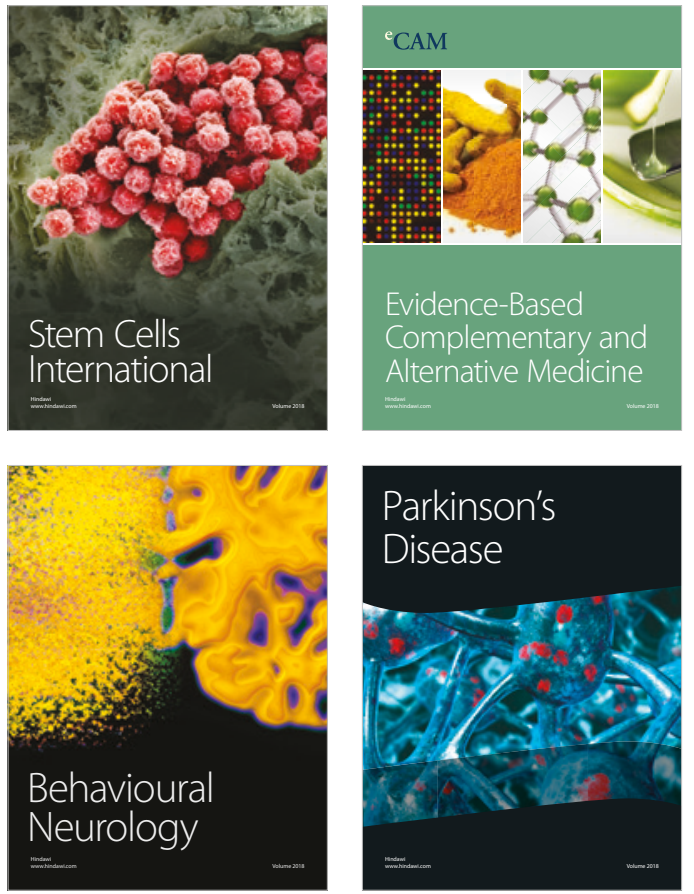

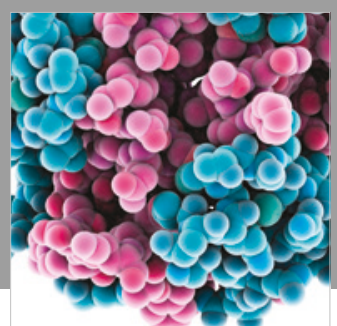

ournal of

Diabetes Research

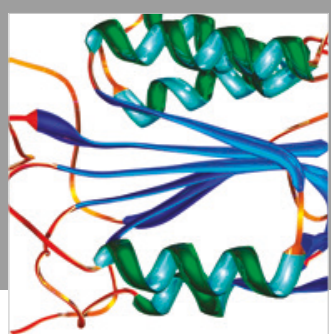

Disease Markers
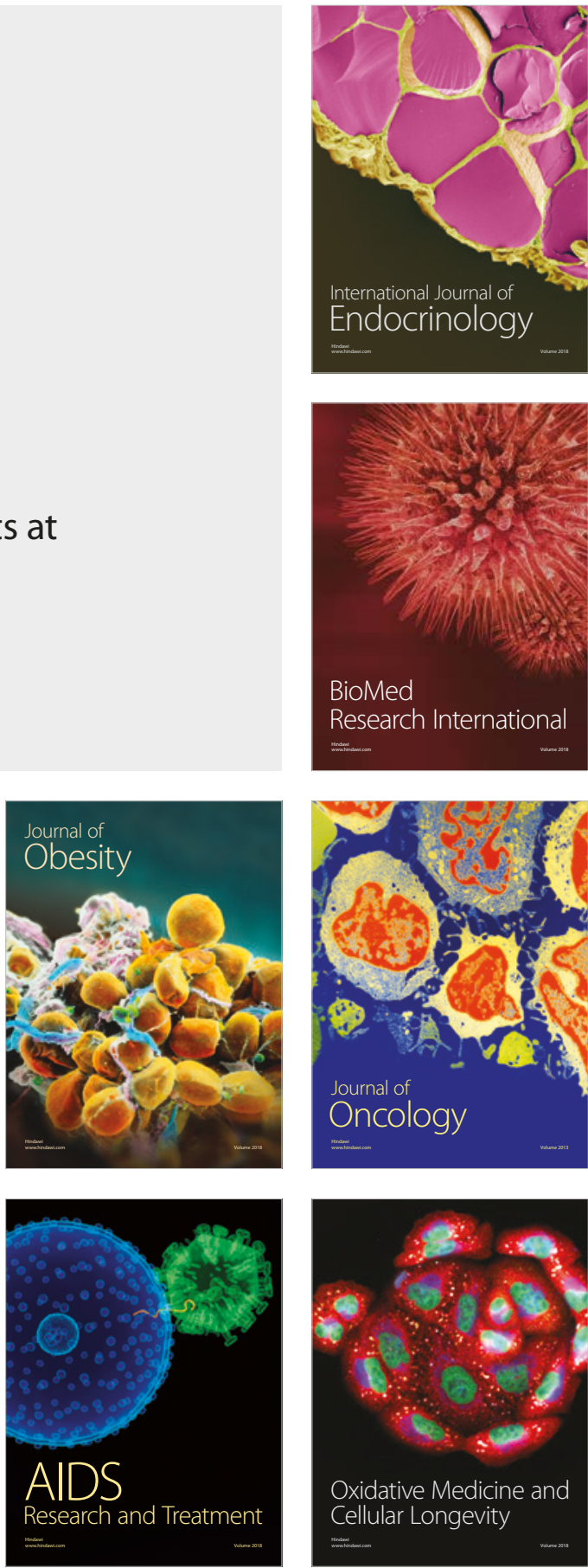